\title{
GOUT: THE KING OF DISEASES AND THE DISEASE OF KINGS
}

\section{Roberto Marcolongo}

\section{Professor of Rheumatology, University of Siena, Italy}

\begin{abstract}
Gout is a metabolic disorder caused by hyperuricemia resulting in joints and other tissues deposition of urate crystals. Clinical manifestation includes recurrent attacks of arthritis and accumulation of crystalline deposits called tophi. Gout is considered a progressive disease that, without long-term treatment, can progress to severe tophaceous gout. The disease is increasing in prevalence as a result of changes in diet, lifestyle and environmental factors. Hyperuricemia has a fundamental role as risk factor in the development of gout and seems also to be a risk factor for some comorbidities. The goal of the treatment of gout is to reduce serum uric acid levels sufficiently that crystals can no longer form. A future research agenda should include the identification of genes associated with risk for gout and the development of new urate lowering drugs with a specific target. In the next future, controlled studies are also required to understand the significance and the underlying mechanism of the association between hyperuricemia and some comorbidities.
\end{abstract}

Key words: Uric acid, hyperuricemia, gout.

\section{INTRODUCTION}

Gout is a metabolic disorder caused by hyperuricemia resulting in deposition of crystals of monosodium urate in joints and other tissues [1]. Clinical manifestations include recurrent attacks of articular and periarticular inflammation, accumulation of articular, osseous, soft tissue and cartilage crystalline deposits, called tophi, interstitial nephropathy with renal function impairment and sometime uric acid calculi in the urinary tract. Deposition of crystals may continue for months or years without causing symptoms until shedding of crystals into synovial fluid triggers the first episode of acute gout.

Gout is considered a progressive disease that, without effective long-term treatment, may progress to severe tophaceous gout with joint damage and significant functional impairment. Much of disability in chronic gout is due to the presence of tophi that may be associated with a destructive deforming arthritis and may ulcerate. The metabolic disorder underlying gout is hyperuricemia which is defined as serum urate concentration more than $7.0 \mathrm{mg} / \mathrm{dl}$ for men and $6.0 \mathrm{mg} / \mathrm{dl}$ for women [1]. Not all subjects with hyperuricemia develop gout and a long period of asymptomatic hyperuricemia may precede the first acute attack. Hence, by itself, hyperuricemia is not sufficient for the expression of gout and asymptomatic hyperuricemia is very common and, in the absence of gout, is not a disease.

\section{HISTORY}

Gout was first identified by the Egyptians in 2640 BC and is one of the oldest recognized diseases [2]. Hippocrates described it as "arthritis of the rich" due to the association with certain foods and excessive alcohol. He believed that gout developed after accumulation of bodily humors, such as "phlegm" which resulted in the painful distension of joints after dietary and sexual excess and a sedentary life. His dictums on diet include advice on the preparation and content of foods, and he recommended moderation, but not abstinence, from wine, "whereby the humors may be kept in healthy balance and disease obviated". Called the "king of diseases" and "disease of kings", gout is perhaps more the disease of plenty. The term "gout", not used until the $13^{\text {th }}$ century, is derived from Latin "gutta" (a drop) which reflects the notion that gout resulted from a local instillation of malevolent humor. These concepts have been ironically illustrated (Figure 1) in paintings of rich persons afflicted with gout. Although many of the advances in the understanding and therapy of gout have emerged in the $20^{\text {th }}$ century, it is worth noting that some

Correspondence to:

Paola Marcolongo

Department of molecular and development medicine, University of Siena, Italy.

Phone: +39.0577.234020

email: paola.marcolongo@unisi.it 
of the fundamental discoveries such as the identification of crystals as urate and the demonstration of urate in tophi, date back to the $17^{\text {th }}$ and $18^{\text {th }}$ centuries. In the $20^{\text {th }}$ century there have been major advances in our knowledge of the etiology of hyperuricemia and the management of gout has been facilitated by the development of many drugs.

The earliest recorded drawings of crystals taken from a tophus are those of Van Leeuwenhoek in 1679 [3]. However, he did not recognize that they were crystals and was unaware of their chemical composition. A century later, Wollaston was able to show that material obtained from a tophus in his own ear was composed of sodium urate.

Elevated levels of uric acid in the serum of patients with gout were demonstrated by Garrod. Crystals of uric acid were seen to form along a linen fiber suspended for 18-48 hours in the acidified serum of gouty patients [4].

\section{OLD THERAPIES}

Hippocrates, in his aphorisms [5], reported that swellings and pains in the joints in most cases are relieved by a local copious affusion of cold water which reduced the swelling and removes the pain. But he also recommended heat and counter irritation. Uncertainty about the best approach to the local treatment of the acute gout persisted for the next centuries [6]. In the first century AD Celsus advocated warm applications according to the intensity of inflammation and advised great caution with regard to cold. Avicenna, in the $11^{\text {th }}$ century, subscribed to this belief and recommended that intractable joints could be cauterized lightly with a hot iron through a layer of salt and oil. Yet earlier Aretaeus the Cappadochian was the first to

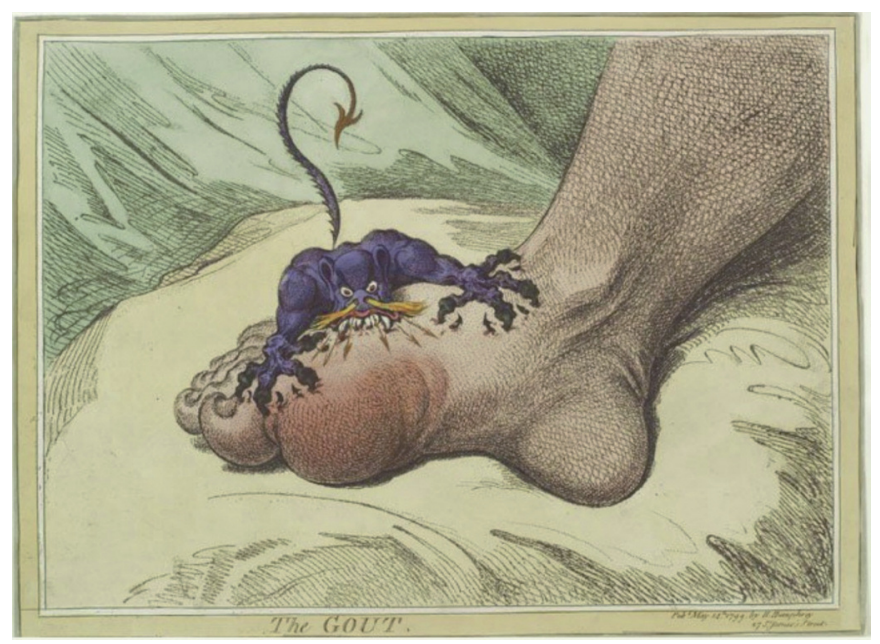

Figure 1. suggest that a specific toxic product or "peccant humor" was the cause of gout and observed that local refrigeration was more helpful than warmth in patients who have "hot species" of arthritis. He asserted the efficiency of cold sea water baths followed by an unction of the joint with oil or a cold poultice of cucumber, lemon peel, plantain leaves and rose petals wrapped in the unscoured wool of a sheep to which a little of rose oil or wine was added periodically. Nevertheless, extreme heat and counter irritation became fashionable for the treatment of acute gout in the $17^{\text {th }}$ century with introduction of the new treatment with moxa. Moxa was a fluffy cotton like plant that was placed on the inflamed joint and ignited. In the $18^{\text {th }}$ century hydrotherapy was introduced in Britain at spas predominantly for the treatment of gout. The history of more targeted and more specific therapies began with the use of colchicum for the treatment of "arthritis" approximately 4000 years ago. Hippocrates advocated powerful purgation with white Hellebore for intractable cases of chronic gout as he believed that the best natural relief for this disease is an attack of dysentery, but there is nothing to suggest that he was aware that it might have more specific value in patients who had acute attacks of gout. In the $6^{\text {th }}$ century AD a byzantine physician, Alexander of Tralles, used Hermodactyl extracted from the corm of Colchicum variegate, a species similar to Colchicum autumnale, as a more selective and specific remedy. Aetius of Amida, another byzantine physician, in the $6^{\text {th }}$ century AD, probably was the first to understand clearly that the therapeutic effect of Hermodactyl were distinct from the gastrointestinal effects.

\section{EPIDEMIOLOGY}

The epidemiology of gout is difficult in determining the precise prevalence and the incidence rate because of the remitting and relapsing nature of the disease, as well as the propensity to misdiagnosis by patients and to a lesser extent by clinicians. The urate concentrations in various populations have been studied extensively. Furthermore, the risk of developing gout at a particular urate concentration has been the subject of several studies. However, the epidemiology of gout is different from that of hyperuricemia. Gout is predominantly a disease of adult men, with a pick incidence in the fifth decade. The disease rarely occurs in men before adolescence or in women before menopause. It is a common disorder that frequently results in significant short-term disability, occupational limitations and utilization of medical services. In 1986, the prevalence of self reported gout in the United States was estimated to be 13.6 per 1000 men and 6.4 per 1000 women [7]. The prevalence has increased over the past few decades in the United States as in other countries that have a 
high standard of living. The study of prevalence in the UK showed that gout is predominantly a disease of older men. Gout becomes more common in women after the menopause $[1,7]$. Multiple genetic and environmental influences prompt the chain of events that regulate uric acid formation, transport and disposal. Among environmental factors modifying serum urate concentration are body weight, diet, lifestyle and social class. An example of the interplay between genetic and environmental factors in determining hyperuricemia is provided by the higher mean serum urate levels among Filipinos living in the United States versus individuals of identical racial background living in the Philippines [8]. The limited ability of members of this population group to excrete uric acid supports the hypothesis that a tendency toward hyperuricemia is inherited and is manifested when a diet with a relatively high purine content, such as the usual American diet, is ingested. The epidemiology of gout in New Zealand is another example that exemplifies genetic and environmental interactions and importance of lifestyle factors in the development of gout. The Maori have marked genetic predisposition to hyperuricemia and gout [9]. However, prior to the $18^{\text {th }}$ century, Maori never experienced gout. Following the imposed extreme changes in diet and lifestyle that followed European settlement, gout appeared in the Maori and showed an increase in prevalence in the $20^{\text {th }}$ century to possibly the highest in the world. In three surveys there was a doubling of prevalence recorded from 1958 to 1992 as well as an increase in proportion of patients with tophi, a younger age of onset of gout and an increase in familial cases. As far as concerns the familial occurrence of gout it is reported in about $20 \%$ of affected patients and hyperuricemia has been demonstrated in one-fourth of firstdegree relatives of gout patients.

\section{GENETICS}

Common primary gout in men often shows strong familial predisposition to gout but the genetic basis remains unknown. Twin studies have shown high heritability for uric acid renal clearance. Recent studies have particularly focused on genes regulating urate transport. The $S L C 22 A 12$ gene codes for human urate transporter 1 (URAT1), a member of the organic anion transporter family that, together with other recently identified transporters, is important in controlling reabsorption of uric acid from the renal tubules. A polymorphism of this gene has been associated with under-excretion of uric acid and hyperuricemia in German Caucasians [10] and a mutation in the gene coding for URAT- 1 has been shown to be protective for development of gout in a Japanese cohort [11]. Furthermore, an association of SLC2A9 [12] with gout has been demonstrated in samples of Maori population.

\section{ASYMPTOMATIC HYPERURICEMIA}

Hyperuricemia alone is seldom a reason for treatment [1]. However hyperuricemia is clearly a risk factor for the development of gout, the risk increasing with a higher urate concentration. Over a period of years moderate hyperuricemia, may lead to gout arthritis, tophus formation and, less commonly, nephrolithiasis. Although there is a little long-term risk of tophus formation in patients with a plasma urate level of $7-8 \mathrm{mg} / \mathrm{dl}$, there is increased risk with higher levels. The causes of hyperuricemia should be determined (renal disease, the use of some drugs, for example) and associated problems such as hypertension, alcoholism, hyperlipidemia or obesity should be demonstrated.

\section{PURINE METABOLISM AND SYNTHESIS OF URIC ACID}

Uric acid is the normal end product of the degradation of purine compounds (Figure 2).

Unlike to the majority of mammals, uric acid is the end product of purine metabolism in humans [1], due to the loss, during the evolution period, of uricase activity which degrades uric acid to more soluble allantoin. The limit of solubility of monosodium urate in plasma is approximately $6.7 \mathrm{mg} / \mathrm{dl}$ at $37^{\circ} \mathrm{C}$. Thus the normal adult mean $( \pm \mathrm{SD})$ serum urate concentration $(5.1 \pm 1.0$ $\mathrm{mg} / \mathrm{dl}$ in men and $4.0 \pm 1.0 \mathrm{mg} / \mathrm{dl}$ in women) provides a narrow margin of safety for urate deposition. In humans, uric acid is derived both from the ingestion of foods containing purines and from the endogenous synthesis of purine nucleotides. The synthesis of

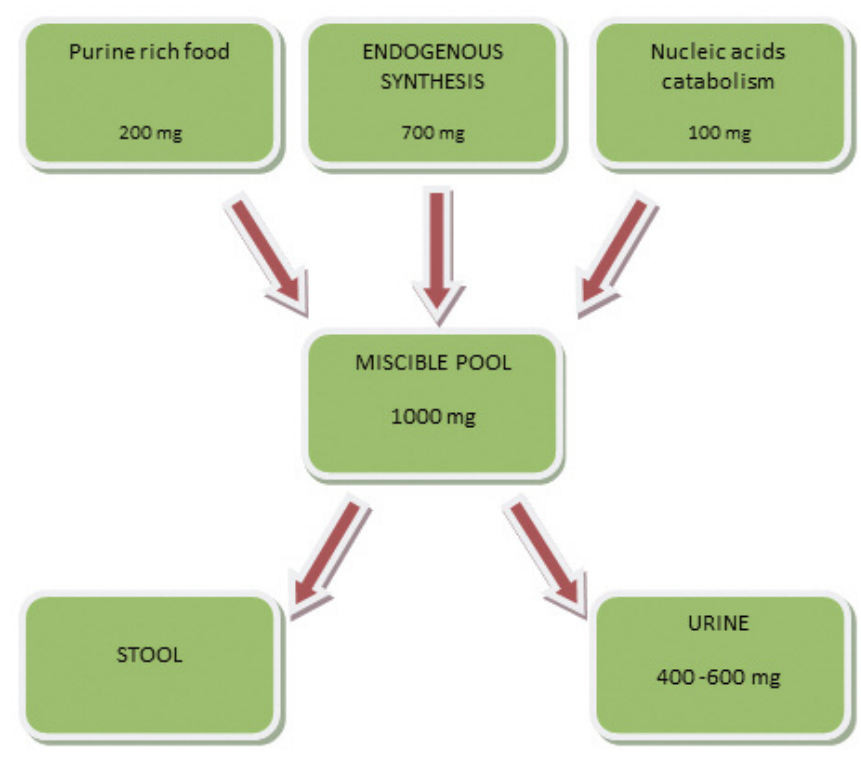

Figure 2. Uric acid metabolism in man. 
purine nucleotides involves biochemical pathways that are closely regulated. In the pathway of purine synthesis de novo, a purine ring is synthesized from small molecule precursors. The total miscible urate pool averages about $1200 \mathrm{mg}$ (range 800-1600) in normal men and about one-half this value in normal women. Since uric acid synthesis averages about $750 \mathrm{mg} /$ day, an estimated two-thirds of the urate pool turns over daily. Gout is invariably characterized by substantial expansion of the uric acid pool and urate supersaturation of the extracellular space [13].

In order to maintain homeostasis, urate must be eliminated from the body as there is no uricase and thus no metabolism of urate in human tissues. Approximately two-thirds of produced urate is eliminated by the kidney with the remainder being excreted into the alimentary tract. The mechanism of urate excretion by the kidney has been studied extensively, although most of the conclusions about renal handling are indirect and derived from alterations caused by administration of some drugs. Almost all of the urate is filtered at the glomerulus. Following filtration there is almost complete reabsorption of urate in the proximal tubule such that only a small amount of the filtered urate passes to the distal tubule.

\section{PATHOGENESIS OF GOUT}

Increased uric acid production and diminished uric acid excretion by the kidney contribute substantially to the hyperuricemia of patients with gout. Thus, patients with hyperuricemia and gout can be sub-classified according to the mechanism responsible for hyperuricemia, overproduction or under-excretion of uric acid or both mechanisms [1].

\section{Urate over-production}

Approximately $10 \%$ of patients with hyperuricemia or gout excrete excessive quantities of uric acid in a 24hour urine collection, commonly defined as more than $800 \mathrm{mg}$ of uric acid excreted while on a normal diet. Isotope labeling studies demonstrated increased rates of uric acid synthesis in such individuals. Inherited derangements in mechanisms regulating purine-nucleotide synthesis account for a very small minority of patients with urate overproduction. The purine synthesis and catabolism is particularly increased in LeschNyhan syndrome due to the Hypoxanthine-guanine phosphoribosyltransferase (HGPRT) deficiency [14]. The syndrome is characterized by high urate production associated with spasticity, choreoathetosis, mental retardation and compulsive self-mutilation.

\section{Urate under-excretion}

Most patients with primary hyperuricemia or gout (more than $90 \%$ ) have a relative deficit in the renal excretion of uric acid. Excretion of normal amounts of uric acid is accomplished in these individuals only when serum urate concentrations are inappropriately high. As previously outlined, virtually all plasma urate is filtered at the glomerulus with more than $95 \%$ of the filtered load undergoing proximal tubular reabsorption. Subsequently, proximal tubular secretion (of about $30 \%$ of the filtered urate load) contributes the major share of excreted uric acid. Post-secretory tubular reabsorption (of about $40 \%$ of the filtered urate load) is another primary mechanism of renal uric acid handling.

\section{Combined overproduction and under-excretion}

Alcohol consumption promotes hyperuricemia by increasing urate production and decreasing uric acid excretion. The higher purine content in some alcoholic beverages is a factor and, in addition, excessive alcohol consumption causes accelerated hepatic breakdown of adenosine triphosphate (ATP) and increased urate production. Furthermore, high alcohol intake may lead to hyperlactic acidemia which blocks uric acid excretion.

\section{Other factors influencing hyperuricemia and gout}

The role of obesity in hyperuricemia is important. In part, excessive dietary purines and alcohol may contribute to the Hyperuricemia [15, 16]. However, obesity per se is also associated with decreased renal excretion of urate. Although all the mechanisms of causation involved are not understood, the renal under-excretion of urate is usually reversible with a weight-reduction diet. Significant improvement in renal excretion of urate may occur with only modest loss of weight. Secondary gout occurs in older subjects and is caused by acquired conditions which affect turnover of nucleic acids or renal excretion of uric acid, such as myeloproliferative disorders, lymphoproliferative disorders, cytotoxic drugs, fructose ingestion and metabolic syndrome. Sex hormones imbalance has been implicated in patients with gout. It has been reported that both men and women with gout have low Folliclestimulating hormone (FSH), Luteinizing hormone ( $\mathrm{LH})$ and beta-estradiol serum levels [17]. It seems most likely that sex hormones primarily influence serum urate concentrations through changes in renal uric acid excretion. Furthermore, experimental studies have indicated that sex hormones may also alter hepatic xanthine oxidase activity, with both oophorectomy and testosterone treatment resulting in increased xanthine oxidase activity. Some other studies hypothesized that a failure of the protein urate-binding capacity resulting in a significant increase of free uric acid are the characteristic of gouty patients and the estrogen must be included among the factors capable of increasing uric acid-plasma proteins binding preventing the risk of deposition of free uric acid in tissues. Indeed, an impaired urate-binding may result in a reduced urate solubility and facilitate urate deposition. 


\section{PATHOGENESIS OF THE ACUTE ATTACK OF GOUT}

Gout was the first disease in which crystals were identified in joint effusions and subsequently incriminated in the pathogenesis of the arthritis. Intra-articular injection of urate crystals was shown to induce gout-like attacks, even in normal subjects. However, the finding of crystals in synovial fluids of asymptomatic joints suggests that factors other than simply the presence of crystals may be important in modulating the inflammatory reaction. The ingress of neutrophils in the joint cavity appears to be the most important event for developing acute urate crystal induced synovitis. Intra-articular neutrophils can be activated both by direct contact with crystals and by exposure to soluble mediators. Urate crystals are direcly able to initiate and sustain intense attacks of acute inflammation because of their capacity to stimulate the release of numerous inflammatory mediators.

The basic pathogenesis in acute gout attack involves the response of polymorphonuclear leukocytes to the formation of urate crystals [1]. Most evidence suggests than an acute attack is associated with the shedding of crystals from a preformed subsynovial formation. Both the protein coating of the crystal and the nature of the responding cell are important to the inflammatory response [18]. Crystals formed in vivo have a protein coat which is necessary for a cellular response. Crystals coated with IgG react with Fc receptors on the surface of the responding cell promoting phagocytosis (Figure 3) with the formation of phagolysosomes. When the phagolysosomal enzymes strip the IgG from the surface of the crystal, the hydrogen bond on the surface of the crystal induce membranolysis of the phagolysosome and cause rupture of cell from within, resulting in the release

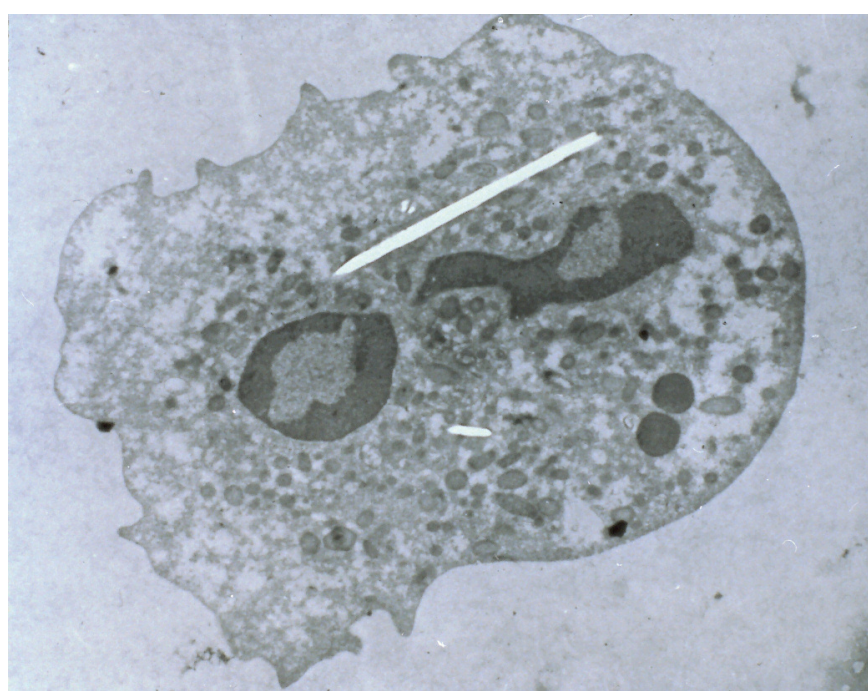

Figure 3. Neutrophil phagocytosis of urate crystals. of a variety of inflammatory mediators . These include mediators such as cyclooxygenase and lipoxygenase metabolites of arachidonic acid, phospholipase A2 activating protein, lysosomal proteases, interleukins IL-l, IL-6 and tumor necrosis factor $\alpha$ (TNF $\alpha$ ) $[19,20]$. In men, the first gout attack occurs between the fourth and sixth decades of life. In women, age at onset is older and varies with several factors, the most important being age of menopause. The onset of a gouty attack is usually heralded by the rapid development of warmth, swelling, erythema and severe pain in the affected joint. Pain varies from moderate to its most intense level over an 8to 12 -hours period. The intensity of pain may vary among the patients. The initial attack is usually monoarticular and in one-half of patients involves the first metatarsophalangeal joint. Other joints that are frequently involved in this early stage are the midfoot, ankles, heels, and knees and less commonly the wrists, fingers and elbows. Gout does not always need to be articular, with involvement of several sites including the olecranon, the bursa and the Achilles tendon. Systemic symptoms such as fever, chills and malaise may accompany acute gout attack due to the systemic release from the gouty joint into the venous circulation of IL-1, IL-6 and TNF $\alpha$ and may help to explain the capacity of the acute gouty attack to affect joints in more than one region. The natural course of untreated acute attack varies from episodes of mild pain that resolve in several hours to severe attacks lasting 1-2 weeks. The acute gouty attack may be self-limited by certain changes in not only the physical properties and proteins coating intra-articular urate crystals, but also in the balance between proand anti-inflammatory factors. However, the precise mechanism of the spontaneous resolution of acute gouty inflammation remains to be defined. The formation of urate crystals is regarded as central to the pathogenesis of gouty arthritis. In most instances, urate crystallizes as a monosodium in oversaturated joint tissues.

As mentioned above, only a minority of individuals with sustained hyperuricemia develops acute arthritis and chronic tophaceous gout. The reasons for this exceptions are poorly understood at present. Some recent studies indicate that there may be important inhibitors of urate crystal growth in synovial fluids and that patients with gout may have reduced inhibitory activity, offering an explanation for the development of gout in some, but not other, hyperuricemic subjects.

The decreased solubility of sodium urate at the lower temperature of peripheral structures such as the toes and ears may help explain why urate crystals deposit in these areas.

A variety of physicochemical factors may influence crystal formation in tissues. A prerequisite is sufficient concentration or supersaturation of uric acid in biological fluids. Given appropriate conditions of low temperature and $\mathrm{pH}$, initial very small crystals may then form. The driving force for such nucleation is a function of the size of the crystals. It is only when a critical size 
is reached that the stability and continuing crystal growth takes place.

Several factors have been recognized as precipitants of acute attacks of gout. These include acute illness, trauma, surgery, alcohol (especially beer and some wines) and drugs that either increase or decrease the plasma urate concentration. The period between attacks when the patients are asymptomatic has been termed intercritical gout [1]. Since many patients with a single attack of gout are either not diagnosed or are overlooked in epidemiological surveys it is not possible to determine the proportion of patients who have subsequent attacks. Nonetheless, it would appear that a significant majority of patients will have more than one attack, with the second attack usually occurring within one or two years. In a small minority of patients many years may elapse between attacks.

\section{Synovial fluid analysis and crystal identification}

A definite diagnosis of gout attack is possible only by aspiration and inspection of either synovial fluid or tophaceous material and the demonstration of characteristic monosodium urate crystals (Figure 4). The crystals are usually needle or rod-shaped. On compensated polarized microscopy they appear as bright, birefringent crystals that are yellow when parallel to the axis of slow vibration on the first order color compensator [2]. The crystals are usually intracellular during acute attacks, but small, truncated extracellular crystals commonly appear as the attack subsides and during the intercritical periods. The synovial fluid findings are consistent with moderate to severe inflammation. The leukocyte count usually falls between 5,000 an 80,000 cells $/ \mathrm{mm}^{3}$ with an average between 15,000 to 20,000 . The cells are predominantly neutrophils [2].

\section{TREATMENT OF ACUTE GOUT ATTACK}

The goal of treating the acute gout attack is to eliminate the pain and other symptoms caused by the intense inflammation. Many agents can be used effectively, including colchicine, nonsteroidal anti-inflammatory drugs (NSAIDs) and corticosteroids [1, 21]. The sooner after onset of an attack that one of these drugs are given, the more rapidly the signs of inflammation will resolve.

\section{Colchicine}

Is an effective agent for the treatment of acute gout and pain subsides within 48 hours in the majority of patients. Colchicine inhibits the polymerization of microtubules of neutrophils by binding their protein subunits and preventing their aggregation. Membrane- dependent functions such as chemotaxis and phagocytosis may, therefore, be disrupted. Colchicine also inhibits the pro- duction of crystal-induced chemotactic factor and IL-6. Most of the adverse effects of colchicine are dose-related and will disappear if a sufficiently low dose is taken. The principal adverse effects are gastrointestinal and usually consist of diarrhea or vomiting. Prolonged high dosage can lead to a malabsorption-syndrome and if sufficiently severe, an hemorrhagic gastroenteritis.

\section{Nonsteroidal anti-inflammatory drugs}

Nonsteroidal anti-inflammatory drugs are probably used more often than colchicine for acute gout. Many physicians prefer to use indomethacin, but other NSAIDs may be just as effective. In general, NSAIDs are started in their recommended maximal doses at the first sign of an attack. The dose may be lowered as symptoms resolve, but medication should be continued until the arthritis has resolved totally for at least 48 hours. When used in this way, the response rate of NSAIDs approaches that of oral colchicine. NSAIDs may cause significant side-effects, most commonly gastrointestinal symptoms. These appear to be less common when NSAIDs are used to treat acute gout compared to their use in other conditions.

\section{Corticosteroids}

Corticosteroids are usually reserved for patients in whom colchicine or NSAIDs are contraindicated or ineffective. There are reports of relapse or early recurrence complicating their use, however recent data noted relapse infrequently. Response time was comparable to that of NSAIDs. For acute gout, 20-40 mg doses of prednisone (or equivalent other corticosteroids) are administered daily for three to four days and then gradually diminished over 1-2 weeks. If oral administration is problematic, the hospitalized patients can be treated by with equivalent doses of intravenous methylprednisolone. Aspiration and intra-articular corticosteroids injection is a useful alternative in the patient who has attack in one or two large joints.

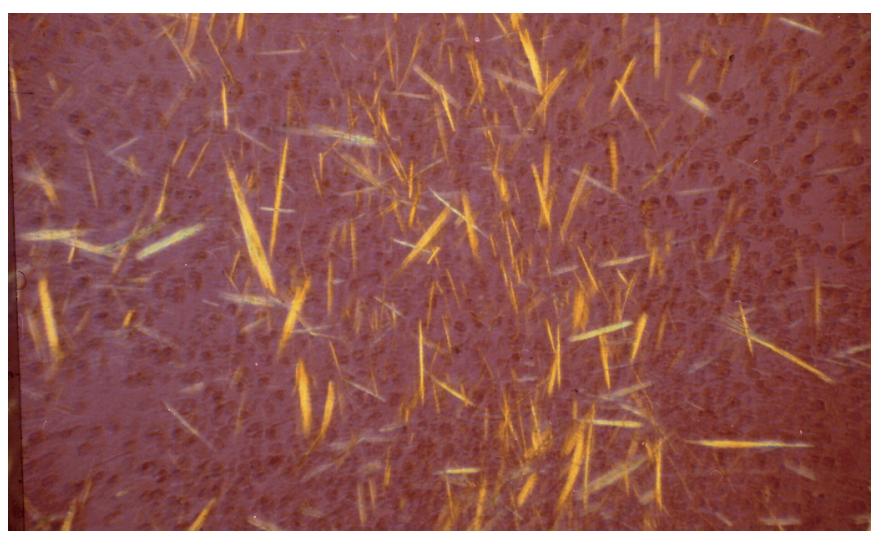

Figure 4. Urate crystals from Marcolongo, R. et al., Le Malattie Reumatiche. Fisiopatologia e Clinica, UTET, Torino, 1991. 


\section{Biological drugs}

The discovery of inflammasome has stimulated the investigation on the efficacy of IL-1 inhibition in acute gout [22]. Three inhibitors have been studied to date, canakinumab (anti-IL-1 monoclonal antibody), kineret (Il-lRa) and rinolacept (IL-1 Trap). Both kineret and rilonacept inhibit IL-1 signaling binding to bioactive IL-1 (both $\alpha$ and $\beta$ forms), thereby inhibiting binding to the IL-1 receptor [23]. In a small case series of patients who presented either intolerance or contraindications to NSAIDs and colchicine preliminary data reported a rapid clinical response.

\section{PROPHYLACTIC TREATMENT OF INTERMITTENT GOUT}

The intercritical periods of acute intermittent gout are as just characteristic of this stage as are the acute attacks $[1,2]$. The previously involved joints are virtually free of symptoms. Despite this, monosodium urate crystals can often be identified in the synovial fluid. Synovial fluids containing crystals also had a higher mean cell count than those with no crystals. These subtle differences may reflect ongoing subclinical inflammation.

Modification of diet, drug regimen and lifestyle may reduce the frequency of acute attacks and render daily drug therapy unnecessary. Weight loss may yield beneficial reduction in serum urate and dietary purine restriction may be an important adjunct to therapy, although plasma urate reduction is usually modest. Alcohol must be avoided because it may both increase production and impair excretion of uric acid. Dehydration and repetitive trauma (as encountered in certain exercises or occupations) may be avoidable causes of gouty attacks. Furthermore, diuretics and other drugs known to contribute to hyperuricemia should be eliminated if possible. The decision to start prophylactic therapy in individual patients depends on issues such as toxicity cost, adherence, and patient tolerance of acute attack. Prophylactic therapy ought not to be considered until one has decided to add an hyperuricemic drug to the regimen. The use of prophylactic colchicine without controlling the hyperuricemia allows tophi to develop without the usual warning signs of acute gouty attacks. The protracted use of small daily doses of colchicine appears to be relatively safe.

\section{CHRONIC TOPHACEOUS GOUT}

This stage of gouty arthritis usually develops after 10 or more years of acute intermittent gout, although patients have been reported with tophi (Figure 5) as their initial clinical manifestation. The transition form from acute intermittent gout to chronic tophaceous gout occurs when the intercritical periods are no longer free of pain, the involved joints are now persistently uncomfortable and swollen, although the intensity of these symptoms is much less than during acute flares. Gouty attacks continue to occur against this painful background, and without therapy they may recur as often as every few weeks $[1,2]$. The amount of background pain also steadily increases with time if appropriate intervention is not started. Gout frequently associates with kidney disease, each being a risk factor for the other. Primary kidney disease can lead to hyperuricemia and it has been suggested that the increasing prevalence of end-stage renal disease may be one cause of recent increases in gout. Kidney damage secondary to gout is associated with urate crystals and microtophi in the interstitium and/or uric acid crystals within tubules. Experimental studies suggest that hyperuricemia may accelerate chronic kidney disease, and several data have demonstrated a significant and independent association between uric acid levels and the progression of chronic kidney disease. Gout is also an independent risk factor for urolithiasis. Uric acid stones occur in $10 \%-20 \%$ percent of all patients with gout. The incidence correlates strongly with the serum urate levels. The likelihood of developing stones reaches $50 \%$ when the serum urate is above $13 \mathrm{mg} / \mathrm{dl}$.

\section{MODIFICATIONS OF ENVIRONMENTAL FACTORS ASSOCIATED WITH THE DEVELOPMENT OF GOUT}

A series of studies clarifying the role of dietary factors have provided new insights into the environmental factors contributing to the development of gout $[15,16]$.

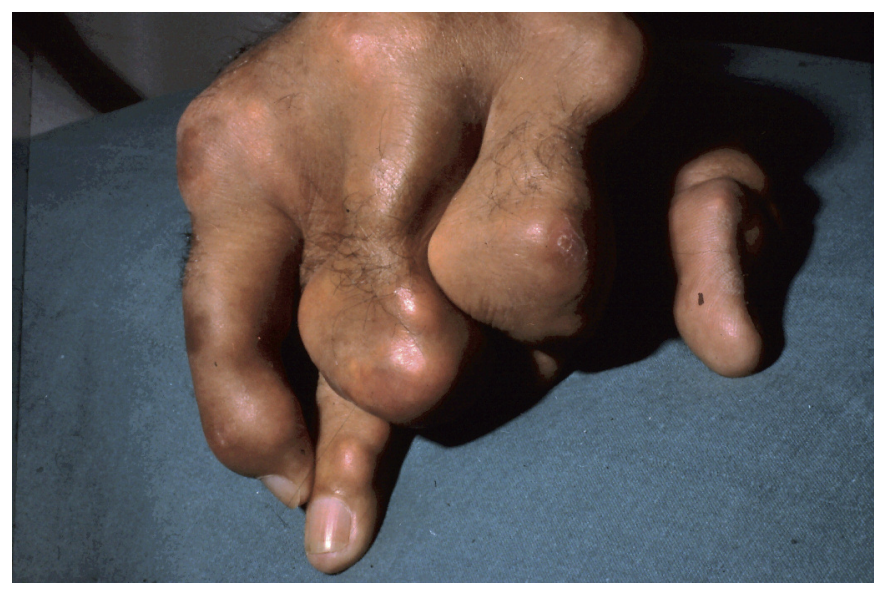

Figure 5. Gout tophi of the hand from Marcolongo, R. et al., Le Malattie Reumatiche. Fisiopatologia e Clinica, UTET, Torino, 1991. 


\section{Diet}

Modification of diet and lifestyle is a core component of gout management. Dietary factors are thought to play a significant role in the increasing prevalence of gout. Despite long-standing links between diet and gout, only recently causative components have been described (high intake of meat and seafood and purinerich vegetables). Traditionally, patients were advised to adopt low purine diets but such diets can rarely be sustained for long and are only occasionally clinically useful. Indeed, dietary purine restriction causes a fail in the plasma of more than $0.5-1.0 \mathrm{mg} / \mathrm{dl}$. On the contrary, alcohol restriction can have a much greater effect on the plasma urate concentration, depending upon the amount consumed. Alcohol raises the serum urate by enhancing urate production and by reducing renal clearance. Acetate conversion to acetyl coenzime A in the alcohol metabolism leads to degradation of adenine nucleotides and accelerated urate production. Furthermore, the metabolism of alcohol to lactic acid results in inhibition of uric acid secretion and a reduction in the fractional clearance of uric acid by the kidney. Consequently, permanent modification of excessive alcohol consumption should contribute significantly to lower the plasma urate concentration. Fructose-rich beverages such as sugar-sweetened soda and orange juice can increase serum uric acid levels and, thus, the risk of gout. Fructose-induced hyperuricemia has been suggested to be related to increased catabolism of preformed purine nucleotides or increased purine synthesis de novo.

\section{Obesity}

The role of obesity in hyperuricemia is important. In part, excessive dietary purines and alcohol may contribute to the hyperuricemia. However, obesity per se is also associated with decreased renal excretion of urate. Although all the mechanisms of causation are not understood, the renal under-excretion of urate is usually reversible even with a modest loss of weight.

\section{Drugs}

Many drugs with either increase serum uric acid levels (e.g. diuretics) or reduced urate levels (e.g. uricosurics drugs) effect these changes via interaction with urate transporters such as URAT 1.

The use of thiazides/diuretics is widely regarded as one of the most common modifiable risk factors for secondary gout, especially in the elderly and in women.

Aspirin has a bimodal effect on urate levels: low doses inhibit uric acid excretion and increase urate levels, while high doses are uricosuric. In elderly patients, aspirin at a dose of $75 \mathrm{mg} /$ day associates with a small but significant increase in serum uric acid. This effect could be significant at a population level because of the widespread use of low-dose aspirin.

\section{URATE-LOWERING AGENTS}

The treatment of hyperuricemia in patients with recurrent or chronic gout implies a long-term commitment to daily therapy and behavioral change. The anti-hyperuricemic agents available are sufficiently potent that dietary restriction of purine is often not necessary, nor is the automatic avoidance of medications that have hyperuricemic properties. The goal of treatment is consistent maintenance of serum urate $<5.0 \mathrm{mg} / \mathrm{dl}$ to allow solubilization of crystalline urate. Appropriate antihyperuricemic agents include uricosuric drugs and xanthine oxidase inhibitors. In either instance. the lowest dose that maintains the serum urate in acceptable ranges should be employed. Because fewer than $10 \%$ of gouty patients are overproducers of uric acid, uricosuric therapy is rarely appropriate. However, because allopurinol is effective in both overproducers and underexcretors and is given in a single daily dose, it is generally recommended and largely used in clinical practice.

\section{Uricosuric agents}

An uricosuric agent is one which lowers the serum urate concentration by increasing the excretion of uric acid by the kidney. This is associated with an increase in the uric acid concentration in the urine. The tendency for uric acid crystal formation would also be reduced by alkalization of the urine. It was with the introduction of treatment with probenecid, sulphinpyrazone and benzbromarone [24] that physicians first were provided with agents that could be used to control hyperuricemia and gout safely and effectively in the majority of patients for long periods of time. Uricosuric agents are effective for patient who have a glomerular filtration rate exceeding $50-60 \mathrm{ml} / \mathrm{min}$, have no history of nephrolithiasis or excessive urine acidity. With advancing age, glomerular filtration rate falls and few patients over 65 years of age can adequately respond to various uricosuric drugs.

\section{Xanthine oxidase inhibitors}

The development of allopurinol led to the most important advance in the treatment of gout and the control of uric acid production. Allopurinol is the agent of choice for patients who have urate overproduction, tophus formation, nephrolithiasis or other controindications to uricosuric therapy. It is the preferred drug in cases of renal insufficiency. Allopurinol reduces serum uric acid by inhibiting xanthine oxidase activity which has been observed to be increased about fourfold in the liver of overproductor gouty patients. Serum and urinary uric acid were reduced dramatically: serum urate levels begin to fall 1 to 2 days after starting treatment with maximal decrements in 1 week. The number of acute attacks of gout was diminished and a gradual resolution of tophi can be observed if the serum urate level is maintained below 5.0 to $6.0 \mathrm{mg} / \mathrm{dl}$. 
Side-effects are represented by dyspepsia, headache, diarrhea and pruritic papula rash that occurs in 3\% to $10 \%$ of the patients. Toxicity can almost always be avoided if dosages are restricted appropriately. Typically, a dosage of $300 \mathrm{mg} /$ day can be started as initial therapy, however, doses of $100 \mathrm{mg}$ are appropriate in the elderly.

Febuxostat is structurally different from allopurinol and lacks the purine ring [23,25]. It is a more selective and potent inhibitor of xanthine oxidase than allopurinol. Doses of $80 \mathrm{mg}$ and $120 \mathrm{mg}$ per day have been shown to be safe and effective in lowering serum uric acid levels and more effective in reducing the size of tophi during 12 months.

\section{Uricolytic drugs}

One potential strategy for managing gout is the use of urate oxidase (uricase) to convert urate to allantoin, a more soluble and easily excreted end product of purine metabolism. Attempts to lower serum uric acid levels using uricase proved that this is an inadequate treatment for chronic hyperuricemia because of its marked immunogenicity and short effective half-life. Recently, it has been developed and tested pegloticase [23], a polyethilene glycol-conjugated form of uricase that minimizes the immunogenicity of the uricase and greatly prolongs the duration of efficacy. Pegloticase was shown to be a very potent form of urate-lowering therapy, reducing plasma urate levels to $<3 \mathrm{mg} / \mathrm{dl}$ in the first 6 hours. The ability of pegloticase to maintain the plasma urate levels below the target of $6.0 \mathrm{mg} / \mathrm{dl}$ depends on the dosage and frequency of the infusions. The most frequent adverse event was the increased incidence of gout flares. This observation highlights the need for more effective antinflammatory prophylaxis as more potent urate-lowering drugs become available.

\section{ASSOCIATION OF GOUT WITH OTHER DISEASES}

Most studies linked hyperuricemia to marked association with several pathologic conditions. The possible association are numerous: uric acid levels seem correlate with hypertension, kidney diseases, cardiovascular diseases, obesity, hyperinsulinemia, hyperleptinemia, hypertriglyceridemia, carotid and coronary artery diseases.

\section{Hypertension}

Hypertension is present in $\mathbf{2 5 - 5 0 \%}$ of gouty patients and $2 \%-4 \%$ of hypertensive patients have gout. Hypertension may be associated with hyperuricemia in three ways: hypertension reduces renal excretion of urate, renal damage for interstitial microtophi may lead to secondary renal hypertension or excessive alcohol consumption may cause both hyperuricemia and hypertension.

\section{Cardiovascular diseases}

A number of epidemiological studies have reported a relation between serum uric acid levels and a wide variety of cardiovascular conditions, including hypertension, metabolic syndrome, coronary artery diseases, cerebrovascular diseases, pre-eclampsia and kidney diseases. However, the relative importance of these associations remain controversial. The main problem is to determine whether uric acid is a risk factor for cardiovascular diseases "independent" of other known risk factors.

\section{Renal diseases}

The only consistent visceral damage caused by hyperuricemia is its effect on the kidney. Several distinct forms of Hyperuricemia-induced renal diseases are recognized, including chronic urate nephropathy, acute uric acid nephropathy and uric acid nephrolithiasis. Progressive renal failure is common in the gouty patients and accounts for up to $10 \%$ of deaths. Hypertension, chronic lead exposure and ischemic heart disease are the most important contributing factors to this complication. However, the role of hyperuricemia as a single factor in chronic parenchymal disease of the kidney remains controversial. Chronic urate nephropathy is a distinct entity that is caused by the deposition of urate crystals in the renal medulla and pyramids and is associated with mild albuminuria.

\section{Obesity}

Hyperuricemia and gout are highly correlated with body weight for both men and women and individuals with gout are commonly overweight compared to the general population. Obesity may be a common factor linking hyperuricemia, hypertension, hyperlipidemia and cardiovascular diseases.

At present, it is very difficult to determine whether uric acid has a causal role in these conditions or whether it is simply a marker for individuals at increased risk. Clinical and epidemiological studies must carefully consider the implications in their choices for adjustment of various risk factors, the method of analysis (particularly when a factor can be both confounder and intermediate), and the outcomes assessed, so as not to miss potentially important upstream effects.

An important question remains: does lowering of uric acid levels through pharmacologic treatment mean improvement of cardiovascular outcomes and mortality? An answer to this question will require well-controlled epidemiological and clinical studies and well-controlled clinical trials.

\section{PROTECTIVE ROLE OF URIC ACID?}

Some studies [26] have argued that hypeuricemia is a good condition to have. Uric acid is the end product 
of purine metabolism in humans due to the loss of uricase activity by various mutations of its gene during the evolution.

Furthermore, $90 \%$ of uric acid filtered by the kidney is reabsorbed instead of being excreted. These facts suggest that evolution and physiology have not treated uric acid as a harmful waste product, but a something beneficial that has to be kept. In the past it has been suggested that hyperuricemia may have a role in intelligence and human performance. Subjects with higher uric acid levels tended to have greater intelligence, achievement-oriented behavior, school performance and reaction time. Other Authors have not confirmed this association. On the other hand, the finding observed is difficult to separate from the eating and social habits associated with economic, cultural and intellectual situations. Furthermore, there is some evidence to support a role for uric acid as a mild neurostimulant. In fact, there is a similarity in chemical structure of uric acid with the trimethylated xanthine caffeine. A second major area of investigation is related to the observation that uric acid can function as an antioxidant that can block superoxide, peroxynitrite and iron catalyzed reactions. It is thought that uric acid contributes to $>50 \%$ of the antioxidant capacity of blood. The ability of hyperuricemia to reduce peroxynitrite-mediated nitrotyrosine formation has been suggested to have a key role in neuroprotection in diseases such as multiple sclerosis, Parkinson's disease, stroke and others. Epidemiological studies suggest that subjects with elevate uric acid levels gave a lower frequency of multiple sclerosis, Parkinson's disease and Alzheimer disease. These studies raise the interesting possibility that use of uric acid or its precursors may have benefit in some neurological diseases.

Other data suggest a potential biological advantage of hyperuricemia in immunosurveillance and generation of adaptive immunity to non-microbial stimuli. A very interesting observation is that there is a negative association between gout and rheumatoid arthritis and probably also between gout and systemic lupus erythematosus suggesting a role of uric acid in the field of autoimmunity.

\section{CONCLUSIONS}

Gout is a very common form of arthritis that increased in prevalence as a result of changes in diet, lifestyle and environmental factors. Symptoms are caused by the deposition of urate crystals in joints and other tissues when there is an excess of urate saturation and when the balance of tissue inhibitors and promoters of crystal formation is in favor of crystallization. The goal of the treatment in gouty patients is to reduce serum uric acid levels sufficiently that crystals can no longer form and that existing crystals are dissolved.
Hyperuricemia has a fundamental role as risk factor in the development of gout and seems also to be an independent risk factor for many comorbidities. While gout itself can be cured by lowering the serum uric acid levels, joint and tissue damage that has already occurred may not be reversible emphasizing the importance of treating the gout before such permanent damage has occurred. A major area of progress should include adequately clinical trials concerning the mechanisms and impact of dietary changes on the development of gout. This will provide important information to recognize patients with gout and those at risk of gout to make appropriate lifestyle changes to reduce the risk of the disease and its complication.

In addition, recent advances in understanding both the genetic and environmental risk factors should facilitate to understand genetic-environment interactions that contribute to the development of hyperuricemia. The research agenda in the next decade will focus, on the development of new urate-lowering drugs that specifically target SLC2A9 and other urate transporters. An important future goal is represented by the identification of genes associated with the risk of gout in those with asymptomatic hyperuricemia, noting that many subjects with hyperuricemia do not develop gout. Our increasing knowledge of the mechanisms of urate transporters and the inflammatory response should lead to the discovery of new improved lowering drugs of serum uric acid. In the next future, accurate controlled studies are also required to understand the significance and the underlying mechanism of the different comorbidities and the true relationships between hyperuricemia and associated diseases.

\section{REFERENCES}

1. Becker, M.A.; Roessler, B. J. Hyperuricemia and gout. Scriver, C. R.; Beaudet, A. L.; Sly, W. S.; Valle, D., eds. The Metabolic Bases of Inherited Disease (7th edition). New-York, McGraw-Hill; 1995: 1655-1677.

2. Klippel, J. H. ed. Primer on the rheumatic diseases (1 $3^{\text {th }}$ edition). New York, Springer; 1998.

3. McCarty, D. J.; A historical note: Leeuwenhoek's description of crystals from a gouty tophus. Arthritis Rheum. 13: 414-418, 1970.

4. Garrod, A. B. The nature and treatment of gout and rheumatic gout. Walton $\theta$ Malberly, London, 1859

5. Marson, P.; Pasero, G. Some historical remarks on microcrystalline arthritis (gout and chondrocalcinosis). Reumatismo. 63(4):199206, 2012

6. Copeman, W. S. C. A short history of gout. Berkeley, University of California Press, 1964.

7. Harris, M. D.; Siegel, L. B.; Alloway, J. A. Gout and Hyperuricemia. Am. Fam. Physician. 59 (4): 925-934, 1999.

8. Healey, L .A.; Skeith, M. D.; Decker, J. L.; Bayani-Sioson, P. S. Hyperuricemia in Filipinos: interaction of heredity and environment. Am. J. Hum. Genet. 19(2): 81-85, 1967.

9. Klemp, P.; Stansfield, S.A.; Castle, B.; Robertson, M.C. Gout is on the increase in New Zealand. Ann. Rheum. Dis. 56(1):22-6, 1997.

10. Graessler, J.; Graessler, A.; Unger, S.; Kopprasch, S; Tausche, A.K.; 
Kuhlisch, E.; Schroeder, H.E. Association of the human urate transporter 1 with reduced renal uric acid excretion and hyperuricemia in a German Caucasian population. Arthritis Rheum. 54(1): 292300, 2006.

11. Kuriki, S.; Okada, R.; Suzuki, K.; Ito, Y.; Morita, E.; Naito, M.; Hamajima, N. SLC22A12 W258X frequency according to serum uric acid level among Japanese health checkup examinees. Nagoya J. Med. Sci. 73(1-2):41-8, 2011.

12. Hollis-Moffatt, J. E.; Xu, X.; Dalbeth, N.; Merriman, M. E.; Topless, R.; Waddell, C.; Gow, P. J.; Harrison, A. A.; Highton, J.; Jones, P. B.; Stamp, L. K.; Merriman, T. R. Role of the urate transporter SLC2A9 gene in susceptibility to gout in New Zealand Maori, $\mathrm{Pa}$ cific Island, and Caucasian case-control sample sets.Arthritis Rheum. 60(11): 3485-3492, 2009.

13. Becker, M.A. Clinical gout and the pathogenesis of hyperuricemia. In: Koopman, W.J. ed. Arthritis and Allied Conditions (14th ed). Philadelphia: Lippincott, Williams and Wilkins; 2001:2281-2313.

14. Segmiller, J. E.; Rosenbloom, F. M.;Kelley, W.M. Enzyme defect associated with a sex linked human neurological disorder and excessive purine synthesis. Science 155: 1682-1684, 1967.

15. Choi, H. K. Diet, alcohol, obesity, hyperuricemia and risk of gout. In: Terkeltaub, R., ed. Gout and other crystal artheropaties, volume 1. Philadelphia: Elsevier Saunders; 2011: 137-147.

16. Singh, J.A.; Reddy, S.G.; Kundukulam, J. Risk factors for gout and prevention: a systematic review of the literature. Curr. Opin. Rheumatol. 2: 192-202, 2011.

17. Rosen, R.; Tomer, Y.; Carel, R.; Weinberger, A. Serum 17-beta-estradiol and testosterone levels in asymptomatic hyperuricaemic men. Clin. Rheumatol. 13(2):219-23, 1994.

18. Popa-Nita, O.; Naccache, P.H. Crystal-induced neutrophil activa- tion. Immunol. Cell Biol. 88(1):32-40, 2010.

19. Busso, N.; So, A. Gout. Mechanisms of inflammation in gout. Arthritis Res. Ther. 2010; 12(2): 206-214, 2010.

20. Chen, C. J.; Shi, Y.; Hearn, A.; Fitzgerald, K.; Golenbock, D.; Reed, G.; Akira, S.; Rock, K. L. MyD88-dependent IL-1 receptor signaling is essential for gouty inflammation stimulated by monosodium urate crystals. J. Clin. Invest. 116(8):2262-2271, 2006.

21. Hamburger, M.; BaraF, H. S.; Adamson, T.C. 3rd; Basile, J.; Bass, L.; Cole, B.; Doghramji, P. P.; Guadagnoli, G. A.; Hamburger, F.; Harford, R.; Lieberman, J. A. 3rd; Mandel, D. R.; Mandelbrot, D. A.; McClain, B. P.; Mizuno, E.; Morton, A. H.; Mount, D.B.; Pope, R. S.; Rosenthal, K. G.; Setoodeh, K.; Skosey, J. L.; Edwards, N. L. Recommendations for the diagnosis and management of gout and hyperuricemia. Phys. Sportsmed. 39(4):98-123, 2011.

22. Kingsbury, S. R.; Conaghan, P. G.; McDermott, M. F. The role of the NLRP3 inflammasome in gout. J. Inflamm. Res. 4: 39-49, 2011.

23. Burns, C. M.; Wortmann, R. L. Gout therapeutics: new drugs for an old disease. Lancet. 8; 377(9760):165-177, 2011.

24. Iwanaga, T.; Kobayashi, D.; Hirayama, M.; Maeda, T.; Tamai, I. Involvement of uric acid transporter in increased renal clearance of the xanthine oxidase inhibitor oxypurinol induced by a uricosuric agent, benzbromarone. Drug Metab. Dispos. 12:1791-1795, 2005.

25. Edwards, N.L. Febuxostat: a new treatment for hyperuricaemia in gout. Rheumatology (Oxford). 48 Suppl 2:ii15-ii19, 2009.

26. Ames, B. N.; Cathcart, R.; Schwiers, E.; Hochstein, P. Uric acid provides an antioxidant defense in humans against oxidant- and radical-caused aging and cancer: a hypothesis. Proc. Natl. Acad. Sci. U S A. 78:6858-6862, 1981. 